This item was submitted to Loughborough's Research Repository by the author.

Items in Figshare are protected by copyright, with all rights reserved, unless otherwise indicated.

\title{
Dispersive hydrodynamics: Preface
}

\section{PLEASE CITE THE PUBLISHED VERSION}

http://dx.doi.org/10.1016/j.physd.2016.07.002

\section{PUBLISHER}

(c) Elsevier

\section{VERSION}

AM (Accepted Manuscript)

\section{PUBLISHER STATEMENT}

This work is made available according to the conditions of the Creative Commons Attribution-NonCommercialNoDerivatives 4.0 International (CC BY-NC-ND 4.0) licence. Full details of this licence are available at: https://creativecommons.org/licenses/by-nc-nd/4.0/

\section{LICENCE}

CC BY-NC-ND 4.0

\section{REPOSITORY RECORD}

Biondini, G., Gennady El, M.A. Hoefer, and P.D. Miller. 2019. "Dispersive Hydrodynamics: Preface”. figshare. https://hdl.handle.net/2134/22195. 


\title{
Dispersive Hydrodynamics: Preface
}

\author{
G. Biondini ${ }^{\mathrm{a}}$, G. A. El ${ }^{\mathrm{b}}$, M. A. Hoeferc ${ }^{\mathrm{c}}$ P. D. Miller ${ }^{\mathrm{d}}$ \\ ${ }^{a}$ State University of New York at Buffalo, Department of Mathematics, Buffalo, NY 14260-2900, USA \\ ${ }^{b}$ Department of Mathematical Sciences, Loughborough University, Loughborough, LE11 3TU, UK \\ ${ }^{c}$ Department of Applied Mathematics, University of Colorado, Boulder, CO 80309, USA \\ ${ }^{d}$ Department of Mathematics, University of Michigan, East Hall, 530 Church St., Ann Arbor, MI 48109, USA
}

\begin{abstract}
This Special Issue on Dispersive Hydrodynamics is dedicated to the memory and work of G. B. Whitham who was one of the pioneers in this field of physical applied mathematics. Some of the papers appearing here are related to work reported on at the workshop "Dispersive Hydrodynamics: The Mathematics of Dispersive Shock Waves and Applications" held in May 2015 at the Banff International Research Station. This Preface provides a broad overview of the field and summaries of the various contributions to the Special Issue, placing them in a unified context.
\end{abstract}

This collection of papers is dedicated to the memory of G.B. Whitham whose profound influence on the theory of nonlinear dispersive waves is difficult to overestimate. Whitham's classic monograph Linear and Nonlinear Waves [1], published in 1974, remains an exceptionally rich and inspiring source of information on the subject, even as the field of nonlinear waves has substantially and further developed over the last several decades.

Many of the papers presented in this Special Issue are co-authored by the participants of the workshop "Dispersive Hydrodynamics: The Mathematics of Dispersive Shock Waves and Applications" that was held May 17-22, 2015 at the Banff International Research Station (BIRS). This meeting brought together some of the leading experts in the areas of dispersive waves, hyperbolic conservation laws, and experimental science with the aim of addressing recent physical and mathematical developments in the field.

Dispersive Hydrodynamics, the main theme of the BIRS workshop and of this Special Issue, is the domain of applied mathematics and physics concerned with fluid motion in which internal friction, e.g., viscosity, is negligible relative to wave dispersion. In conservative systems such as those modeling certain superfluids, light waves in optical materials, and water waves, nonlinearity has the tendency to engender wavebreaking that is mitigated by dispersion. Relevant mathematical models are often hyperbolic systems of partial differential equations with conservative, dispersive corrections that play a fundamental role in the dynamics. Generically, the result of the combined action of nonlinearity and dispersion is a multiscale, unsteady, coherent wave structure called a dispersive shock wave (DSW). In

Email addresses: biondini@buffalo.edu (G. Biondini), g.el@lboro.ac.uk (G. A. El), hoefer@colorado.edu (M. A. Hoefer), millerpd@umich.edu (P. D. Miller) this connection, one should mention the important works of Benjamin and Lighthill [2], Sagdeev [3], and Ostrovsky [4] who studied the oscillatory structure of "collisionless shocks" described by steady, traveling-wave solutions of dissipative-dispersive equations. These early works could be viewed as precursors to the modern understanding of DSWs as fundamental, purely conservative, "superfluidic" unsteady nonlinear wave phenomena.

A DSW is an expanding, modulated nonlinear wavetrain connecting two disparate states, the dispersive analogue of a dissipative, classical shock. Generally, the generation of DSWs represents a universal mechanism to resolve hydrodynamic singularities in dispersive media. Their fundamental role in such media is similar to that of viscous shock waves in classical gas and fluid dynamics. At the same time, DSWs are sharply distinct from their wellstudied dissipative counterparts both in terms of physical significance and mathematical description. Physical manifestations of DSWs include undular bores on shallow water and in the atmosphere (the Morning Glory) as well as nonlinear diffraction patterns in laser and atom (matter wave) optics.

Additionally, the notion of turbulence in traditional, dissipative fluid dynamics can be extended to dispersive hydrodynamic systems and is often referred to as "integrable turbulence" [5]. In this context, turbulence is usually associated with a complex, spatio-temporal ensemble of waves/solitons that requires a statistical description. This emerging theory bridges the notions of integrability and stochasticity, encompassing both weak (wave) and strong (soliton) turbulence, and is a natural extension of deterministic DSW dynamics.

As an area of applied mathematics, dispersive hydrodynamics has origins in soliton theory, conservation laws, and fluid dynamics. In 1965, just over fifty years ago, the seminal computational work of Zabusky and Kruskal 
[6] demonstrated the existence of soliton solutions to the Korteweg-de Vries (KdV) equation through a process of nonlinear wavebreaking. That same year, Whitham introduced a general asymptotic approach to study modulated periodic nonlinear dispersive waves [7, 8]. Both of these contributions considered conservative, nonlinear, dispersive wave problems. Another touchstone appearing in 1965 was Glimm's fundamental work on hyperbolic conservation laws [9] which contributed to the rapid growth in understanding of this field, see, e.g. [10]. The marriage of dispersive nonlinear waves and hyperbolic conservation laws in the context of dispersive hydrodynamics was initiated in 1974 at the hands of Gurevich and Pitaevskii [11] through their study of a Riemann problem for the $\mathrm{KdV}$ equation where the resulting DSWs were understood utilizing Whitham's modulation equations. A multiphase extension of Whitham theory for the $\mathrm{KdV}$ equation was developed in 1980 by Flaschka, Forest and McLaughlin [12] and revealed the deep connection between modulation theory and the integrable structure of $\mathrm{KdV}$ via the spectrum of finite-gap potentials. Shortly thereafter, Lax, Levermore, and Venakides $[13,14]$ showed that exactly the same "multiphase" Whitham equations describe the weak, zero dispersion limit of the KdV evolution. Finally, a very general approach to the description of dispersive regularization of shocks in integrable equations with weak dispersion has become available in the nonlinear steepest descent method of Deift and Zhou [15], which is designed to handle singular limits of Riemann-Hilbert problems such as those arising from inverse scattering. This approach has been applied to the $\mathrm{KdV}$ equation [16] and has been extended to other integrable equations, in particular to the focusing nonlinear Schrödinger (NLS) equation [17].

The Whitham modulation equations for KdV are now known to be strictly hyperbolic and genuinely nonlinear [24], highlighting deep connections between conservation laws and dispersive nonlinear waves. Another important connection between dispersive hydrodynamics and the theory of integrable hydrodynamic type systems [18] has been facilitated by Tsarëv's discovery of the generalized hodograph transform [19] and Krichever's algebro-geometric construction [20] of multiphase waves. More recently, Dubrov universality conjectures [21] introduced a new direction in the rigorous treatment of the initial stage of DSW formation. Additional progress in the understanding of solutions to the Riemann problem for non-integrable nonlinear dispersive wave equations $[22,23]$ has made possible the analytical description of DSWs in more physically-relevant model equations.

An essential aspect of the subject of dispersive hydrodynamics is its physical or experimental realization. Laboratory measurements of DSWs were undertaken in the context of undular bores in shallow water waves by Favre in 1935 [25]. Applications to collisionless plasmas in the 1960s motivated several early theoretical works $[6,11]$. There has been steady interest in geophysical applications, which include surface and internal waves in the ocean and atmosphere (see e.g., [26, 27, 28]). More recently, experiments in ultracold atomic physics $[29,30]$ and nonlinear photonics [31, 32] have inspired the further mathematical study of dispersive hydrodynamics.

The collection of papers presented in this Special Issue consists of 25 articles, which can be roughly grouped into the following sections:

G. B. Whitham memoir and review articles: The memorial paper [33] by Minzoni and Smyth presents a short biography of G. B. Whitham along with a description of his seminal contributions to the theory of nonlinear dispersive waves. The memorial paper is followed by two review articles, both devoted to DSWs.

El and Hoefer [34] present a broad review of DSW theory for integrable and non-integrable nonlinear wave equations from the perspective of Whitham's modulation theory. Detailed descriptions of DSWs for the KdV and defocusing NLS equations are presented following Gurevich and Pitaevskii's matching regularization procedure [11]. The paper then proceeds through more recent developments related to DSWs in non-integrable systems, nonclassical DSWs in dispersively modified non-convex conservation laws and steady oblique 2D DSWs forming from supersonic flows of superfluids past obstacles. Significant attention is paid to DSWs in physical applications, from shallow-water waves to nonlinear optics and Bose-Einstein condensates.

Miller's review article [35] concerns the mathematically rigorous study of DSWs in integrable systems, based on the use of the inverse-scattering transform (IST). Many features of the analysis required to study weakly dispersive nonlinear waves are first explained in the context of the linear Schrödinger equation and its solution by Fourier transforms and Green's functions. The paper then turns to the subject of the defocusing NLS equation, explaining first the weak (average) asymptotics of DSWs by means of LaxLevermore theory, and then using the Deift-Zhou method to obtain strong (locally uniform) asymptotics. These analytical techniques actually provide the correct implicit solution of the modulation equations, yielding a strong coninnection with Whitham's modulation theory. The paper concludes with a section on the notion of universality for dispersive waves and a short description of some newer areas of research.

Whitham equations and dispersive shock waves - theoretical aspects: The paper by Ablowitz, Demirci and $\mathrm{Ma}$ [36] enters the almost uncharted area of twodimensional (2D) DSWs. DSWs arising in the KadomtsevPetviashvili (KP) and 2D Benjamin-Ono (BO) equations are considered for step-like initial data along a parabolic front. Employing a parabolic similarity reduction, the authors reduce the original $\mathrm{KP}$ and $2 \mathrm{D} \mathrm{BO}$ equations to the cylindrical KdV and cylindrical BO equations, respectively. The DSWs in these equations are then studied using Whitham modulation theory and the results are favor- 
ably compared with numerical simulations of DSWs in the original equations.

The paper [37] by Kamchatnov describes the derivation of the modulation equations for a general class of perturbed KdV equations using Whitham's original method, as described in the foundational 1965 paper [7]. The author demonstrates an important difference between the effects of gradient and non-gradient perturbations and proposes a method to eliminate gradient perturbations. This is an extention of the author's generalized modulation theory [38], previously developed to handle non-conservative perturbations.

In [39], Ratliff and Bridges develop modulation theory for a class of systems that lead to hyperbolic equations with degenerate characteristics. They show that, on a slower time scale, the modulation equations exhibiting such degeneracy universally morph into the two-way, dispersive Boussinesq equation. The authors' method provides insight into the Kelvin-Helmholtz instability. Another implication of the method is that the two-way Boussinesq equation is invalid as a model for water waves.

Exact and asymptotic methods for nonlinear waves - rigorous theory: In the paper [40] by Biondini, Fagerstrom and Prinari, the IST is used to solve the defocusing NLS equation with fully asymmetric non-zero boundary conditions. In contrast to the case of symmetric non-zero boundary conditions, which can be effectively treated by making use of a uniformization variable, in the asymmetric case, the direct and inverse scattering problems can be successfully formulated on a single sheet of the spectral variable, notwithstanding the square root branch cut of the asymptotic eigenvalues. It is also shown that no pure soliton solutions, i.e., reflectionless potentials, exist in the asymmetric case.

In [41], Deng, Biondini and Trillo study the small dispersion limit of the $\mathrm{KdV}$ equation with periodic initial conditions and apply the results to the Zabusky-Kruskal experiment. The WKB method is employed to obtain an asymptotic expansion of the scattering eigenfunctions, which in turn yield an asymptotic expression for the trace of the monodromy matrix. Such expression is then analyzed to characterize the asymptotic properties of the scattering problem. The results, which show excellent agreement with numerical simulations of the scattering problem, imply that, in the limit of zero dispersion, the problem gives rise to a pure soliton gas.

The paper [42] by Dyachenko, D. Zakharov and V. Zakharov is devoted to the construction of a novel class of potentials of the Schrödinger equation, termed primitive potentials. These potentials are shown to generate a broad class of bounded, non-vanishing aperiodic solutions of the $\mathrm{KdV}$ hierarchy. The authors interpret these solutions as an example of integrable turbulence in the framework of the KdV equation.

In [43], Dubrovin, Grava and Klein study the formation of $2 \mathrm{D}$ DSWs in solutions to the generalized KP equation.
The main result of the paper is a numerically supported conjecture about the universal asymptotic description of the solution to the generalized KP equation for generic initial data. The description is made in terms of a special solution to the second equation of the Painlevé-I hierarchy. This conjecture extends universality theory for critical behavior in Hamiltonian equations with one space dimension to $2 \mathrm{D}$ problems.

The paper [44] by Tovbis and El explores interrelations between Whitham modulation theory for the focusing NLS equation and the rigorous Riemann-Hilbert problem approach to the description of rapidly oscillating solutions that develop in the evolution of the focusing NLS equation with small dispersion. Understanding the links between the two major approaches in the theory of nonlinear dispersive waves could prove beneficial for a broad range of problems involving the semiclassical focusing NLS equation.

In [45], Miller and Wetzel use IST methods to study the Benjamin-Ono equation in the small-dispersion limit. The authors begin with a complete, recently-developed theory for the relevant nonlocal, direct scattering problem valid for rational initial data. They then show explicitly how the scattering data behaves asymptotically as the dispersion parameter tends to zero. In particular, the authors rigorously determine the asymptotic location of the soliton eigenvalues, the number of which grows without bound in the small-dispersion limit.

Fluid dynamics applications: In [46], Grimshaw and Yuan study the propagation of internal ocean waves and, in particular, how DSWs (undular bores) are modified by variable bottom topography in the framework of the variable-coefficient $\mathrm{KdV}$ equation. Numerical simulations and asymptotic analysis based on Whitham modulation equations are used to investigate the effect of a polarity change. This change occurs for certain topography profiles when the undular bore passes through a critical point at which the coefficient of the quadratic nonlinear term in the $\mathrm{KdV}$ equation changes sign.

In [47], Khusnutdinova and Zhang undertake numerical modeling of weakly nonlinear surface and interfacial ring waves in a two-layer fluid within the framework of the recently derived $2+1$-dimensional concentric KdV-type equation. The $2 \mathrm{D}$ version of the dam-break problem is studied and the obtained numerical solutions are shown to exhibit the formation of concentric DSWs. The effect of a piecewise-constant shear flow is also discussed.

In [48], Kurkina, Rouvinskaya, Talipova, Kurkin and E. Pelinovsky, motivated by the shoaling propagation of internal tidal waves, perform a numerical study of the nonlinear disintegration of a sine wave in the framework of the Gardner (extended KdV) equation containing both quadratic and cubic nonlinear terms. The authors observe the formation of multiple undular bores at intermediate times and study their properties. The evolution of a sine wave is shown to strongly depend on the relative signs of 
the quadratic and cubic terms in the Gardner equation.

In [49], Milewski and Wang perform high resolution computations of capillary gravity wave packets from primitive fluid equations. Self-focusing dynamics of patches of ripples are observed and compared to solutions of the (critical) focusing 2D NLS equation. The authors discuss similarities and differences to the focusing of light beams in Kerr media and explore long time dynamics - beyond the regime of validity of NLS.

The paper [50] by Kalisch, Khorsand and Mitsotakis presents a systematic derivation of balance laws for the Serre-Green-Naghdi (SGN) equations, a Boussinesq-type system describing fully nonlinear dispersive shallow water waves. Numerical solutions of the SGN equations are constructed via a high-order finite element method and are used to study the energy balance in shallow water undular bores and shoaling solitary waves.

In [51], Camassa, Marzuola, Ogrosky and Vaughn study dissipative-dispersive traveling waves for a model of gravitydriven film flows in cylindrical domains. The authors explore the mean thickness threshold for traveling wave formation for viscous films and compare the results with experiments.

Ostrovsky and Stepanyants [52] study the interaction of a $\mathrm{KdV}$ soliton with a long wave in a rotating ocean within the framework of the rotation-modified KdV equation, also known as the Ostrovsky equation. A model dynamical system describing the interaction is derived and studied analytically and numerically. It is shown that solitons riding on long waves can propagate over great distances in a rotating ocean.

The paper [53] by Trillo, Klein, Clauss and Onorato reports the observation of surface gravity DSWs developing from initial depressions in an experiment conducted in a shallow water tank. The results of the experiment are shown to be in excellent agreement with numerical simulations of DSWs for "time-like" versions of the KdV equation and the (nonlocal) Whitham equation.

Other applications: In [54] Gershenzon, Bambakidis and Skinner use the Frenkel-Kontorova model and its continuum limit, the sine-Gordon equation, to develop a mathematical model of macroscopic non-lubricant friction. The model is based on solutions of the sine-Gordon modulation (Whitham) equations and connects the kinetic and dynamic parameters of the frictional process. The application of the model to the description of seismic events over a wide range of rupture and slip velocities is discussed.

The paper [55] by Giglio, Landolfi and Moro, develops an integrable extended model for van der Waals fluids via the theory of nonlinear conservation laws and the description of phase transitions in terms of classical (dissipative) shock waves. The authors propose a novel approach to the construction of multiparameter generalizations of the van der Waals model and provide a detailed comparison of their extended model with well-known empirical models. Possible further generalizations are discussed that could associate thermodynamic phase transitions with DSW formation.

Smyth [56] studies the Riemann dam break problem for a system of equations describing optical beam propagation in nematic liquid crystals. This system is comprised of the defocusing NLS equation coupled with an elliptic equation describing the non-local response of the medium. In the regime of strong nonlocality, the dispersive dynamics induced by small initial jumps is shown to be described by the $\mathrm{KdV}$ equation exhibiting bright solitons despite the defocusing nature of the associated NLS equation. The modulation DSW solution of the $\mathrm{KdV}$ equation is then used to explain solution features observed in numerical simulations. The generation of a linear, highly oscillatory wavetrain propagating ahead of the DSW is observed and its length is estimated using phase- and group-velocity arguments.

Incoherent nonlinear dispersive waves: The paper [57] by Xu, Garnier, Faccio, Trillo and Picozzi reports a unified presentation of different forms of incoherent shock waves that emerge in the long-range interaction regime of a turbulent optical wave system described by a nonlocal NLS equation. Some of the incoherent wave dynamics presented in the paper are shown to exhibit DSWs when considered in the spectral (frequency) domain.

The paper [58] by Randoux, Walczak, Onorato and Suret is an experimental and numerical study of nonlinear propagation of random waves in optical fiber systems accurately described by the integrable one-dimensional NLS equation. Statistical properties of incoherent waves are examined. Heavy- and low-tailed deviations from Gaussian statistics are observed in the focusing and defocusing regimes respectively. Heavy-tailed statistics in the focusing regime are associated with the formation of rogue waves. The phenomenon of intermittency is revealed in the integrable turbulence of random optical waves.

\section{Acknowledgements}

We are grateful to Tim Sauer, the Overseeing Editor of Physica D, for his help and support throughout the preparation of this Special Issue. We thank the Banff International Research Station for providing an exceptional environment for the workshop "Dispersive Hydrodynamics: The Mathematics of Dispersive Shock Waves and Applications", May 17-22, 2015. Finally, we would like to thank the authors and reviewers for their outstanding contributions to this Special Issue.

[1] G. B. Whitham, Linear and nonlinear waves, Wiley, New York, 1974.

[2] T. B. Benjamin, M. J. Lighthill, On cnoidal waves and bores, Proc. Roy. Soc. Lond. A 224 (1159) (1954) 448-460.

[3] R. Z. Sagdeev, Cooperative phenomena and shock waves in collisionless plasmas, Reviews of Plasma Physics, 4 (1966) 23.

[4] L. A. Ostrovskii, Envelope shock waves, Sov. Phys. JETP 27(4) (1968) $660-664$.

[5] V. E. Zakharov, Turbulence in integrable systems, Studies in Applied Mathematics, 122 (2009) 219 - 234. 
[6] N. J. Zabusky and M. D. Kruskal, Interaction of 'solitons' in a collisionless plasma and the recurrence of initial states, Physical Review Letters, 15 (1965) 240.

[7] G. B. Whitham, Non-linear dispersive waves, Proc. Roy. Soc. London A 283 (1965) 238-261.

[8] G. B. Whitham, A general approach to linear and non-linear dispersive waves using a Lagrangian, J. Fluid Mech. 22 (02) (1965) 273-283.

[9] J. Glimm, Solutions in the large for nonlinear hyperbolic systems of equations, Comm. Pure Appl. Math., 18 (1965) 697715 .

[10] P. D. Lax, Hyperbolic systems of conservation laws and the mathematical theory of shock waves, SIAM, Philadelphia, 1973.

[11] A. V. Gurevich and L. P. Pitaevskii, Nonstationary structure of a collisionless shock wave, Soviet Physics JETP 38 (1974) $291-297$.

[12] H. Flaschka, M. G. Forest, and D. W. McLaughlin, Multiphase averaging and the inverse spectral solution of the Korteweg-de Vries equation, Communications on Pure and Applied Mathematics 33 (1980) 739-784.

[13] P. D. Lax and C. D. Levermore, The small dispersion limit of the Korteweg-de Vries equation: 1-3, Communications on Pure and Applied Mathematics 36 (1983) 253-290, 571-593, 809-830.

[14] S. Venakides, The zero-dispersion limit of the Korteweg-de Vries equation with non-trivial reflection coefficient, Communications on Pure and Applied Mathematics 38 (1985) 125-155.

[15] P. Deift, X. Zhou, A steepest descent method for oscillatory Riemann-Hilbert problems, Bull. Am. Math. Soc. 26 (1) (1992) 119-123.

[16] P. Deift, S. Venakides, X. Zhou, New results in small dispersion $\mathrm{KdV}$ by an extension of the steepest descent method for Riemann-Hilbert problems, Int. Math. Res. Notices 1997 (6) (1997) 285-299.

[17] S. Kamvissis, K. T. -R. McLaughlin, P. D. Miller, Semiclassical soliton ensembles for the focusing nonlinear Schrödinger equation, Annals of Math. Studies, 154. Princeton University Press, Princeton, N.J., 2003.

[18] B. A. Dubrovin, S. P. Novikov, Hydrodynamics of weakly deformed soliton lattices. Differential geometry and Hamiltonian theory, Russian Mathematical Surveys 44 (6) (1989) 35-124.

[19] S. P. Tsarev, Poisson brackets and one-dimensional Hamiltonian systems of hydrodynamic type, Sov. Math. Dokl. 31 (1985) 488-491.

[20] I. M. Krichever, Method of averaging for two-dimensional "integrable" equations, Func. Anal. Appl. 22 (3) (1989) 200-213.

[21] B. Dubrovin, On Hamiltonian perturbations of hyperbolic systems of conservation laws, II: Universality of critical behavior, Comm. Math. Phys., 267 (2006) 117-139.

[22] G. A. El, Resolution of a shock in hyperbolic systems modified by weak dispersion, Chaos 15 (2005) 037103.

[23] M. A. Hoefer, Shock waves in dispersive Eulerian fluids, J. Nonlinear Sci. 24 (3) (2014) 525-577.

[24] D. Levermore, The hyperbolic nature of the zero dispersion $\mathrm{KdV}$ limit, Communications in Partial Differential Equations 13 (1988) 495-514.

[25] H. Favre, Étude théorique et experimentale des ondes de translation dans les canaux découverts, Dunod, Paris, 1935.

[26] N. F. Smyth, P. E. Holloway, Hydraulic jump and undular bore formation on a shelf break, J. Phys. Oceanogr. 18 (7) (1988) 947-962.

[27] H. Chanson, Current knowledge in hydraulic jumps and related phenomena. A survey of experimental results, Euro. J. Mech. B 28 (2) (2009) 191-210.

[28] D. R. Christie, The morning glory of the Gulf of Carpentaria: a paradigm for non-linear waves in the lower atmosphere, Austral. Met. Mag. 41 (1992) 21-60.

[29] Z. Dutton, M. Budde, C. Slowe, and L. V. Hau, Observation of quantum shock waves created with ultra-compressed slow light pulses in a Bose-Einstein condensate, Science, 293 (2001)
663.

[30] M. A. Hoefer, M. J. Ablowitz, I. Coddington, E. A. Cornell, P. Engels, and V. Schweikhard, Dispersive and classical shock waves in Bose-Einstein condensates and gas dynamics, Physical Review A 74 (2006) 023623.

[31] W. Wan, S. Jia, and J. W. Fleischer, Dispersive superfluidlike shock waves in nonlinear optics, Nature Physics, 3 (2007) 46-51.

[32] C. Conti, A. Fratalocchi, M. Peccianti, G. Ruocco, and S. Trillo, Observation of a gradient catastrophe generating solitons, Physical Review Letters, 102 (2009) 083902.

[33] T. A. Minzoni, N. F. Smyth, Modulation theory, dispersive shock waves and Gerald Beresford Whitham, Physica D, this issue.

[34] G. A. El, M. A. Hoefer, Dispersive shock waves and modulation theory, Physica D, this issue.

[35] P. D. Miller, On the generation of dispersive shock waves, Physica D, this issue.

[36] M. J. Ablowitz, A. Demirci, Y-P. Ma, Dispersive shock waves in the Kadomtsev-Petviashvili and two dimensional BenjaminOno equations, Physica D, this issue.

[37] A. M. Kamchatnov, Whitham theory for perturbed Korteweg - de Vries equation, Physica D, this issue.

[38] A. M. Kamchatnov, On Whitham theory for perturbed integrable equations, Physica D, 188 (2004) 247.

[39] D. J. Ratliff, T. J. Bridges, Whitham modulation equations, coalescing characteristics, and dispersive Boussinesq dynamics, Physica D, this issue.

[40] G. Biondini, E. Fagerstrom, B. Prinari, Inverse scattering transform for the defocusing nonlinear Schrödinger equation with fully asymmetric non-zero boundary conditions, Physica $\mathrm{D}$, this issue.

[41] G. Deng, G. Biondini, S.Trillo, Small dispersion limit of the Korteweg - deVries equation with periodic initial conditions and analytical description of the Zabusky-Kruskal experiment, Physica D, this issue.

[42] S. Dyachenko, D. Zakharov, V. Zakharov, Primitive potentials and bounded solutions of the $\mathrm{KdV}$ equation, Physica D, this issue.

[43] B. Dubrovin, T. Grava, C. Klein, On critical behaviour in generalized Kadomtsev - Petviashvili equations, Physica D, this issue.

[44] A. Tovbis, G. A. El, Semiclassical limit of the focusing NLS: Whitham equations and the Riemann-Hilbert Problem approach, Physica D, this issue.

[45] P. D. Miller, A. N. Wetzel, The scattering transform for the Benjamin-Ono equation in the small-dispersion limit, Physica $\mathrm{D}$, this issue.

[46] R. Grimshaw, C. Yuan, The propagation of internal undular bores over variable topography, Physica D, this issue.

[47] K.R. Khusnutdinova, X. Zhang, Nonlinear ring waves in a twolayer fluid, Physica D, this issue.

[48] O. Kurkina, E. Rouvinskaya, T. Talipova, A. Kurkin, E. Pelinovsky, Nonlinear disintegration of sine wave in the framework of the Gardner equation, Physica D, this issue.

[49] P.A. Milewski and Z. Wang, Self-focusing dynamics of patches of ripples, Physica D, this issue.

[50] H. Kalisch, Z. Khorsand, D. Mitsotakis, Mechanical balance laws for fully nonlinear and weakly dispersive water waves, Physica D, this issue.

[51] R. Camassa, J. Marzuola, H. R. Ogrosky, N. Vaughn, Traveling waves for a model of gravity-driven film flows in cylindrical domains, Physica D, this issue.

[52] L. A. Ostrovsky, Y. A. Stepanyants, Interactions of solitons with long waves in a rotating fluid, Physica D, this issue.

[53] S. Trillo, M. Klein, G. Clauss, M. Onorato, Observation of dispersive shock waves developing from initial depressions in shallow water, Physica D, this issue.

[54] N. I. Gershenzon, G. Bambakidis, T. E. Skinner, SineGordon modulation solutions: application to macroscopic nonlubricant friction, Physica D, this issue. 
[55] F. Giglio, G. Landolfi, A. Moro, Integrable extended van der Waals model, Physica D, this issue.

[56] N. F. Smyth, Dispersive shock waves in nematic liquid crystals, Physica D, this issue.

[57] G. Xu, J. Garnier, D. Faccio, S. Trillo, A. Picozzi, Incoherent shock waves in long-range optical turbulence, Physica D, this issue.

[58] S. Randoux, P. Walczak, M. Onorato, P. Suret, Nonlinear random optical waves: integrable turbulence, rogue waves and intermittency, Physica D, this issue. 